\title{
Long-term follow up of Hodgkin lymphoma
}

Research Paper

\author{
David Perez-Callejo ${ }^{1, *}$, Lorea Zurutuza ${ }^{1, *}$, Ana Royuela ${ }^{2}$, Maria Torrente ${ }^{1}$, Beatriz \\ Núñez ${ }^{1}$, Virginia Calvo ${ }^{1}$, Miriam Méndez ${ }^{1}$, Fernando Franco ${ }^{1}$, María Auxiliadora \\ Brenes $^{1}$, Juan Cristobal Sánchez ${ }^{1}$ and Mariano Provencio ${ }^{1}$ \\ ${ }^{1}$ Medical Oncology Department, Hospital Universitario Puerta de Hierro-Majadahonda, Madrid, Spain \\ ${ }^{2}$ Statistics Department, Hospital Universitario Puerta de Hierro-Majadahonda, Madrid, Spain \\ *These authors contributed equally to this work \\ Correspondence to: Mariano Provencio, email: mariano.provencio@salud.madrid.org \\ Keywords: Hodgkin lymphoma; survival; standardized mortality ratio \\ Received: September 27, $2017 \quad$ Accepted: January 13, $2018 \quad$ Published: February 03, 2018 \\ Copyright: Perez-Callejo et al. This is an open-access article distributed under the terms of the Creative Commons Attribution \\ License 3.0 (CC BY 3.0), which permits unrestricted use, distribution, and reproduction in any medium, provided the original author \\ and source are credited.
}

\section{ABSTRACT}

Background: Hodgkin lymphoma (HL) is the paradigm of curable disease. This study analyzed the overall survival (OS) of patients with $\mathrm{HL}$ and compared their survival between decades and with the expected survival of a general population.

Results: The median follow-up was 22 years. The median os was 33 years. The incidence mortality rate for all causes is 2 per every 100 patients per year. The OS of our cohort at 10 years from diagnosis was 76\% (95\% CI: $72-79$ ) and $52 \%$ at 30 years (95\% CI: 48-57). Overall SMR (1980-2013) was 2,943 (95\% CI: 2,518-3,439). Excluding the primary tumor as the cause of death, the SMR obtained is 2,266 (95\% CI: 1,895-2,710). The SMR for those patients diagnosed before the year 2000 was 2,097 (95\% CI: 1,732-2,539); and for those diagnosed after 2000 was 5,218 (95\% CI: 8,655). The group of patients diagnosed after 2000 had statistically significant more advanced stages, were older and less responsive to treatment.

Conclusions: Despite the advances achieved, the risk of death remains higher than in the general population, mainly for those patients diagnosed after year 2000, even after almost $\mathbf{4 0}$ years of follow-up. This data might suggest a shift to more aggressive forms of disease in recent years.

Patients and methods: A total of 595 patients diagnosed with $\mathbf{H L}$ were included between January 1966 and February 2014. The standardized mortality ratio (SMR) was analyzed using the annual rate of mortality in the general Spanish population, adjusted for age, sex and time period.

\section{INTRODUCTION}

Hodgkin lymphoma (HL) is the paradigm of curable disease within the field of Medical Oncology, along with germ cell tumors. Globally, 65950 new cases of HL are diagnosed each year, accounting for $0.5 \%$ of all cancer diagnoses [1]. It is therefore considered a rare disease. In Spain, this disease has an incidence rate higher than that of the world population (2.1 and 2.5 cases per 100,000 persons-year in women and men, respectively, in Spain, compared to 0.7 and 1.1 cases worldwide) [2]. Besides, the current Spanish trend is that new diagnoses are increasing
(12.5-14.7\% more), according to the Spanish Network of Cancer Registries (REDECAN) [3].

In recent decades, due to the improvement in diagnostic techniques, advances in molecular biology and in the development of therapeutic options, the survival of these patients has been significantly increased [4]. This fact leads to the need to monitor the occurrence of late complications, such as the appearance of second tumors or cardiovascular events, with a consequent impact on the morbidity and mortality of these patients [5]. However, there is little information regarding the impact of new treatments regarding late toxicities, adapted to the patient's 
risk, with less extension in the radiotherapy field and shorter chemotherapy schemes.

The main objective of this study was to analyze the overall survival (OS) of patients with HL and to compare it with the expected survival in the general Spanish population, with a long follow-up and search for a possible improvement in survival in the last decades of treatment, and whether there has been any change in long-term morbidity and mortality.

\section{RESULTS}

\section{Patient characteristics and survival analysis}

Out of a total of 624 patients diagnosed with HL, 29 patients diagnosed prior to 1975 were excluded due to a lack of epidemiological population data prior to that date. Patient characteristics are described in Table 1.

The median follow-up was 22 years (p25-p75: 8-30 years) for the entire cohort and 29 years (p25-p75: 17-33 years) for disease-free alive patients.

The median OS was 33 years. The incidence mortality rate for all causes is 2 per every 100 patients per year. The OS of our cohort at 10 years was $76 \%$ (95\% CI: 72-79), at 20 years 66\% (95\% CI: 62-70), at 30 years $52 \%$ (95\% CI: $48-57)$ and at 40 years $42 \%(95 \%$ CI: 36-49\%) (Figure 1). The PFS at 10 years was 63\% (95\% CI: 58-67), at 20 years 55\% (95\% CI: 51-60), at 30 years $46 \%$ (95\% CI: $41-51)$, and at 40 years $39 \%$ (95\% CI: 33-45\%) (Figure 2). The DFS for those patients with a complete response at 10 years was 64\% (95\% CI: 60-68), at 20 years $57 \%$ (95\% CI: 52-61), at 30 years 48\%: 43-52) and at 40 years 40\% (95\% CI: 34-47\%) (Figure 3)

\section{Comparison between mortality rates by study group and general population}

SMR was used to quantify the increase or decrease in mortality of the study cohort compared to the general Spanish population. Overall SMR, including all-cause death, was 2,943 (95\% CI: 2,518-3,439), indicating that patients with HL triple on average the risk of death compared to the general population.

Stratifying by sex, women had a higher mortality rate than men, with an SMR of 4,220 (95\% CI: 3,119- 5,584), compared to 2,590 (95\% CI: 2,147-3,125), respectively (Figure 4). Regarding the analysis of the different age groups at diagnosis, those patients belonging to the younger age group ( $\leq 30$ years) presented a significantly higher SMR than the other groups, with an SMR of 9,653 (95\% CI: 7,555-12,333). In patients between 31 and 59 years of age, SMR was 2,560 (95\% CI: 1,996-3,283) and in the population over 60 years of age it was 1,400 (95\% CI: 0.991-1.980), finding no differences in mortality compared to the general population.
Regarding the analysis according to the year to the diagnosis, for those patients with HL diagnosed before the year 2000, the SMR was 2,698 (95\% CI: 2,282-3,190); while for patients diagnosed after the year 2000 it was 7,195 (95\% CI: 4,691-11,035).

Excluding the primary tumor as the cause of death, the SMR obtained is 2,266 (95\% CI: 1,895-2,710). If the cohort is divided according to the year of diagnosis of HL, the SMR for those diagnosed before the year 2000 was 2,097 (95\% CI: 1,732-2,539); and for those diagnosed after 2000 was 5,218 (95\% CI: 3,146-8,655).

\section{Adjusted analysis}

A multivariable Cox regression was performed to jointly assess the association of gender, age at diagnosis, and year of diagnosis (before and after the year 2000) with all-cause mortality in our cohort. Results are shown in Table 2.

The variables associated with all-cause mortality were sex (in males, $\mathrm{HR}=1.33,95 \%$ CI 1.03-1.73, $p=0.030)$ and being over 60 years old (HR with respect to under 30 years $=4.38 ; \% 2.98-6.43, p<0.001$ ).

The characteristics of the patients were analyzed according to the year of diagnosis in order to find an explanation for the SMR of the patients diagnosed after the year 2000, along with a worse prognosis observed within our cohort. The group of patients diagnosed after 2000 $(n=96)$ had more advanced stages $(92 \%$ presented stages II + III + IV vs $80 \%$ in the group $<2000, p=0.010)$, were older $(17 \% \geq 60(p<0.001)$ and were less responsive to treatment (no complete response $20 \%$ vs $5 \%$ in the $<2000$ group, $p<0.001$ ) (Table 3 ).

\section{DISCUSSION}

In Oncology, HL is usually presented as an example of curable disease due to its high number of DFS [6]. However, long-term survival studies have described that patients with HL do not have the same mortality compared to the general population, adjusted for age and sex [7]. In routine clinical practice, patient follow-up is focused on the early detection of complications such as the development of second malignancies, cardiac toxicities or other toxicities associated to treatment, which are commonly the cause of death [8].

In 1999, Provencio et al. [9] demonstrated an increased risk of mortality in patients with HL compared to the general Spanish population, 20 years after diagnosis.

In this study, we reported that even after almost 40 years from diagnosis of HL, patients triple the risk of death from all causes, compared to the general population. When studying the different causes of death, only $22.76 \%$ of them were due to the lymphoma itself. Therefore, the excess death observed in these patients is mainly due to other causes, primely second tumors, fatal 
Table 1: Patient demographics

\begin{tabular}{|c|c|c|}
\hline Characteristics & Valid $N$ & $N(\%)$ \\
\hline Sex & 594 & \\
\hline Male & & $366(61.62)$ \\
\hline Female & & $228(38.38)$ \\
\hline Age at diagnosis (years) & 590 & \\
\hline$\leq 30$ years & & $308(52.20)$ \\
\hline $31-59$ years & & $238(40.34)$ \\
\hline$\geq 60$ years & & $44(7.46)$ \\
\hline Year of diagnosis & 591 & \\
\hline$<2000$ & & $495(83.76)$ \\
\hline$\geq 2000$ & & $96(16.24)$ \\
\hline Ann Arbor stage & 585 & \\
\hline I & & $103(17.61)$ \\
\hline II & & $269(45.98)$ \\
\hline III & & $137(23.41)$ \\
\hline IV & & $76(12.99)$ \\
\hline Hodgkin lymphoma subtypes & 589 & \\
\hline Nodular sclerosis (NS) & & $271(46.01)$ \\
\hline Mixed cellularity (MC) & & $196(33.28)$ \\
\hline Lymphocyte-rich (LR) & & $41(6.96)$ \\
\hline Lymphocyte depleted (LD) & & $51(8.66)$ \\
\hline Non-Specified (NS) & & $30(5.09)$ \\
\hline Response to treatment & 573 & \\
\hline Complete & & $532(92.84)$ \\
\hline Non complete & & $41(7.16)$ \\
\hline Relapse & 558 & \\
\hline Yes & & $174(31.18)$ \\
\hline No & & $384(68.82)$ \\
\hline Secondary tumor development & 516 & \\
\hline Yes & & $106(20.54)$ \\
\hline No & & $410(79.46)$ \\
\hline Exitus & 591 & \\
\hline Yes & & $259(43.82)$ \\
\hline No & & $332(56.18)$ \\
\hline Cause of death & 246 & \\
\hline Primary tumor & & $56(22.76)$ \\
\hline Secondary tumor & & $56(22.76)$ \\
\hline Other causes & & $134(54.47)$ \\
\hline
\end{tabular}

cardiac events or other causes not related to the primary tumor). It is postulated that the risk of developing second malignancies is mainly due to complications derived from radiotherapeutic treatment used in HL, age, smoking history, and recently, it has been associated with a genetic predisposition $[10,11]$.

The risk of death was significantly higher in women than in men. However, in the internal analysis of our cohort, it was the men who had a higher risk of mortality by any cause, mainly due to presenting more advanced disease subtypes (lymphocyte depletion) at the time of diagnosis. Also of note, the great mortality of patients under 30 years of age (eleven-fold; SMR of 11.14), which could be justified by the fact that in the general population of the same age, the risk of death is very low, so a fatal event in our cohort would have a greater impact on the difference between observed and expected events.

In addition, and despite the advances achieved in the treatment of HL in the last decade, the risk of death was even greater in patients diagnosed after 2000. To 


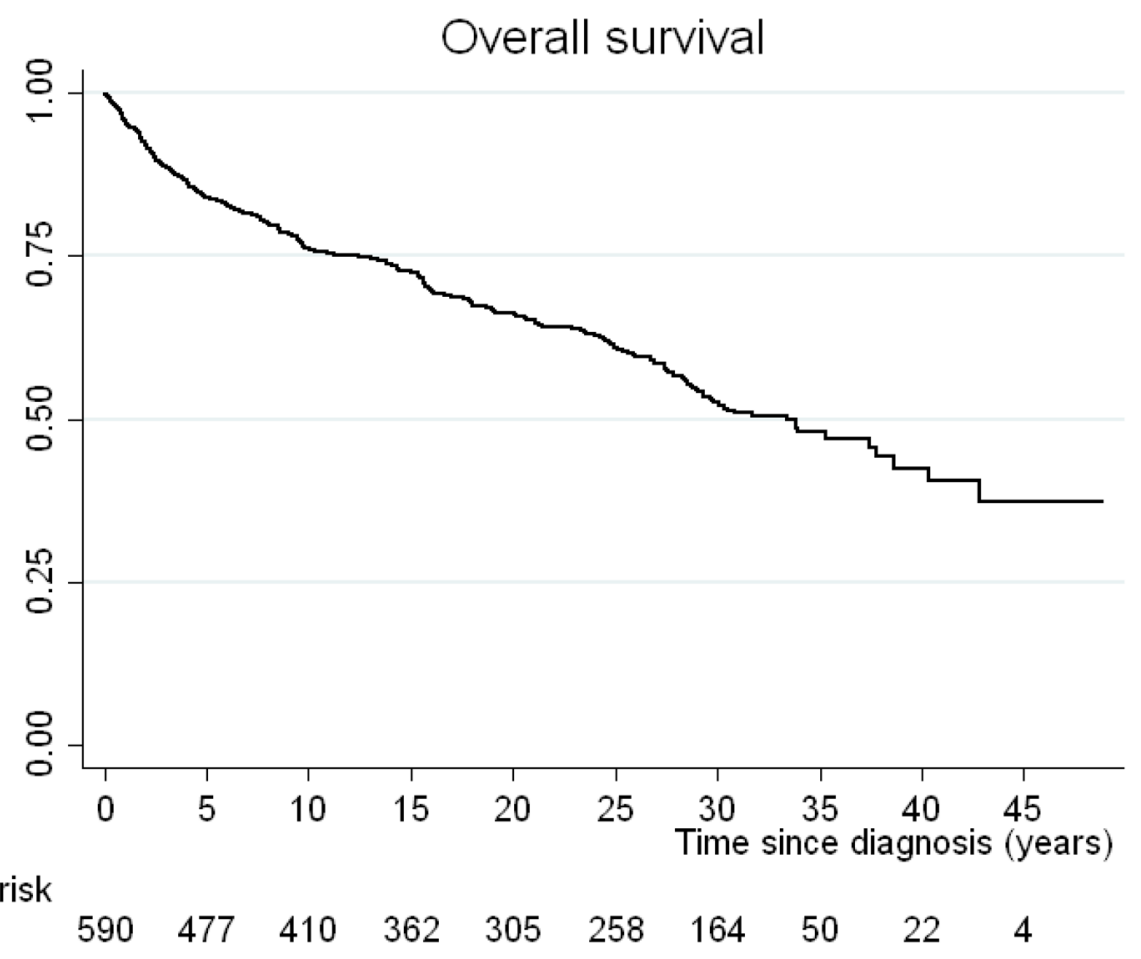

Figure 1: Kaplan-Meier curve for overall survival (OS) of the cohort.

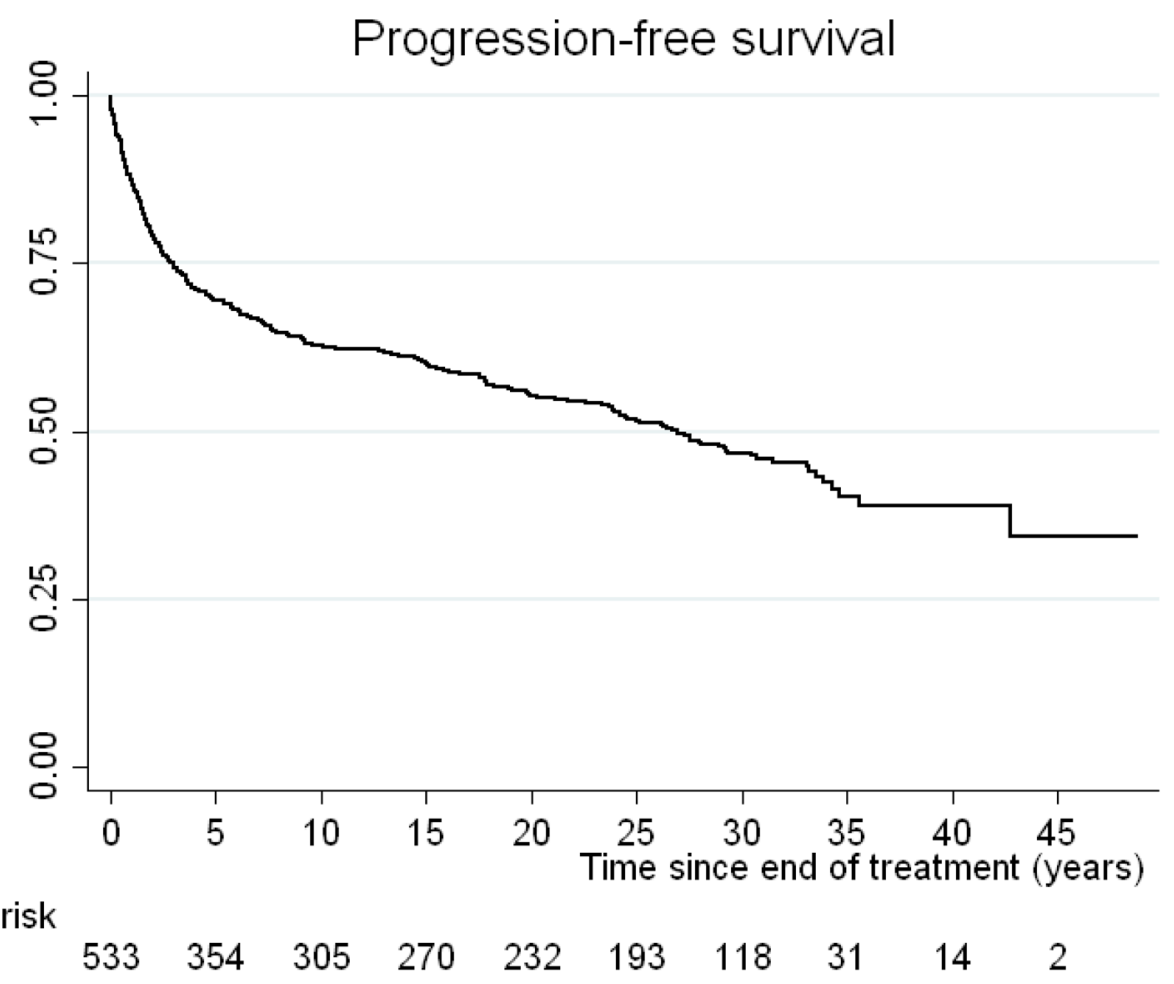

Figure 2: Kaplan-Meier curve for progression-free survival (PFS) of our cohort. 
understand the differences in mortality in relation to the time of diagnosis (before and after 2000), we compared both subgroups in our cohort. Patients diagnosed after the year 2000 had a worse prognosis, since there was a higher proportion of patients with advanced age (16.67\% diagnosed after 2000 vs $5.67 \%$ diagnosed before 2000), and additionally had lower rates of complete response to initial treatment $(20.25 \%$ vs $5.07 \%$, respectively), both with statistical significance.

Therefore, we can conclude that in the Spanish population, HL currently presents a tendency towards more aggressive forms, with older age patients and a

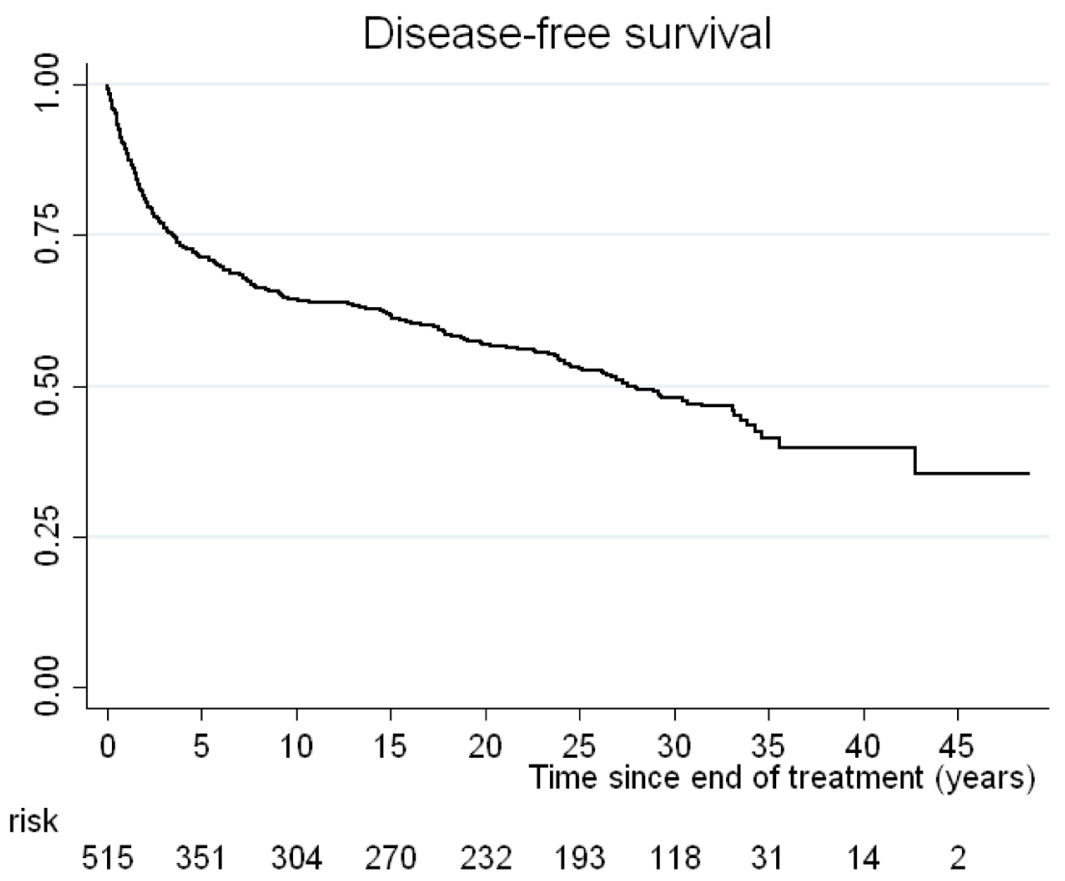

Figure 3: Kaplan-Meier curve for disease-free survival (DFS) of those patients with a complete response after first line treatments.

Number at risk

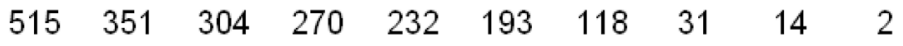

$\operatorname{SMR}(95 \% \mathrm{Cl})$

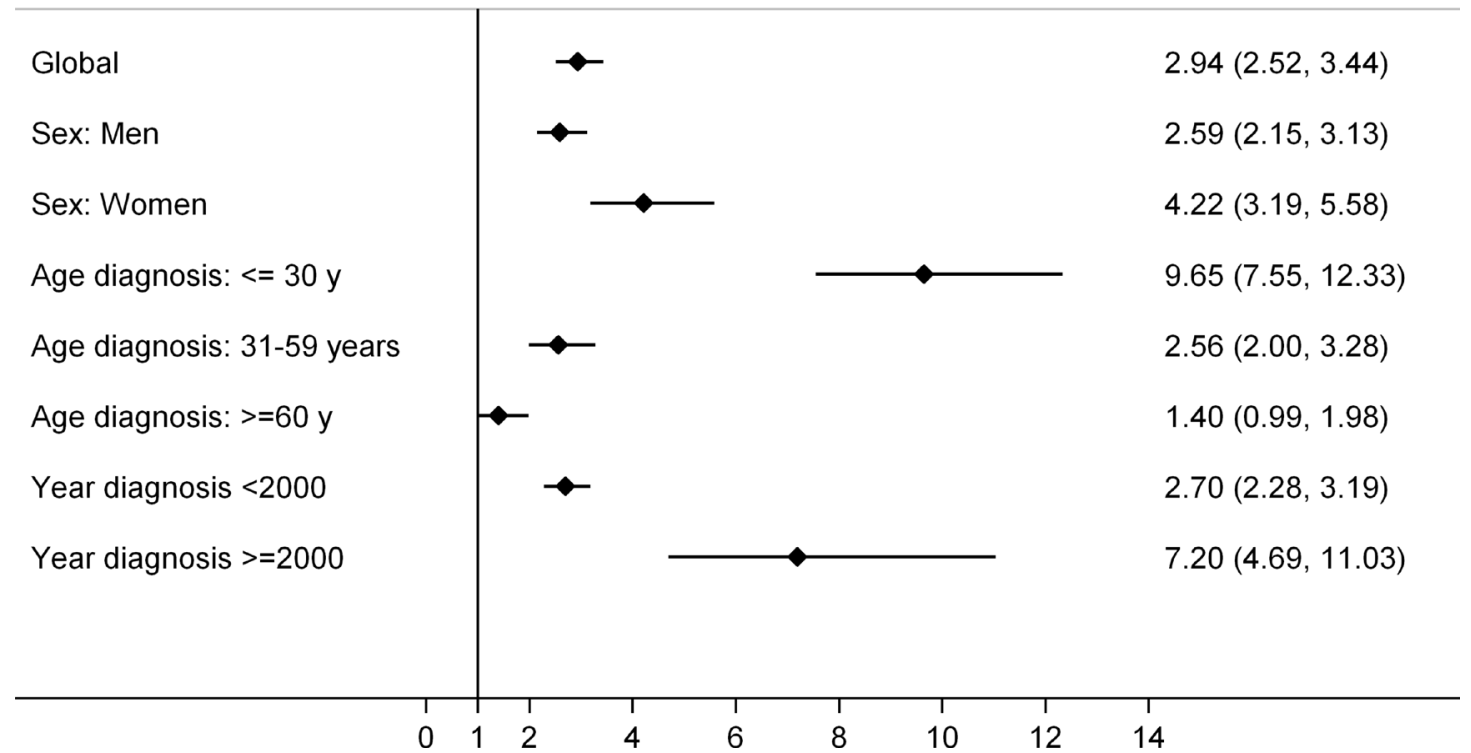

Figure 4: Forest plot of standardized mortality ratios (SMR), global and by subgroups. The forest plot was generated through "Distiller SR Forest Plot Generator from Evidence Partners". 
Table 2: Multivariable Cox regression for all-cause mortality

\begin{tabular}{lcc}
\hline Variables & HR (CI 95\%) & $\boldsymbol{p}$ \\
\hline Sex (male) & $1.335(1.028-1.732)$ & 0.030 \\
Age at diagnosis & & --- \\
$\quad \leq 30$ years & Ref. cat. & 0.136 \\
$\quad 31-59$ years & $1.224(0.938-1.596)$ & $<0.001$ \\
$\quad \geq 60$ years & $4.380(2.983-6.430)$ & 0.359 \\
Year of diagnosis $(\geq 2000)$ & $1.225(0.794-1.889)$ & \\
\hline
\end{tabular}

HR: Hazard Ratio; CI: Confidence Interval.

Table 3: Patient characteristics according to the year of diagnosis $(<2000 \mathrm{vs} \geq 2000)$

\begin{tabular}{|c|c|c|c|}
\hline \multirow{2}{*}{ Characteristic } & $<2000$ & $\geq 2000$ & \multirow{2}{*}{$p$} \\
\hline & $N(\%)$ & $N(\%)$ & \\
\hline Sex & & & 0.626 \\
\hline Male & $307(62.02)$ & $57(59.38)$ & \\
\hline Female & $188(37.98)$ & $39(40.63)$ & \\
\hline Ann Arbor stage & & & 0.081 \\
\hline I & $96(19.39)$ & $7(7.95)$ & \\
\hline II & $223(45.05)$ & $45(51.14)$ & \\
\hline III & $113(22.83)$ & $23(26.14)$ & \\
\hline IV & $63(12.73)$ & $13(14.77)$ & \\
\hline Age at diagnosis (years) & & & 0.001 \\
\hline$\leq 30$ & $267(54.05)$ & $41(42.71)$ & \\
\hline $31-59$ & $199(40.28)$ & $39(40.63)$ & \\
\hline$\geq 60$ & $28(5.67)$ & $16(16.67)$ & \\
\hline Response to treatment & & & 0.000 \\
\hline Complete & $468(94.93)$ & $63(79.75)$ & \\
\hline Non complete & $25(5.07)$ & $16(20.25)$ & \\
\hline Exitus & & & 0.000 \\
\hline Yes & $232(47.15)$ & $26(27.08)$ & \\
\hline No & $260(52.85)$ & $70(72.92)$ & \\
\hline Cause of death & & & 0.061 \\
\hline Primary tumor & $48(21.62)$ & $8(34.78)$ & \\
\hline Secondary tumor & $55(24.67)$ & $1(4.35)$ & \\
\hline Other causes & $119(53.60)$ & $14(60.87)$ & \\
\hline
\end{tabular}

lower rate of complete response to first line treatments. There may be a bias regarding the transfer of complex patients to third-level hospitals such as ours and this may not represent the real situation of the global and current change in this lymphoma. In all, there may be an evident improvement in the average life expectancy in the Western population in the last decades and perhaps this could explain the differences in the risk of death. This is verified with a similar rate of OS at 20 years compared to that of other recently published series, which is around 65\% [4].

The findings observed in this study may be of great relevance in the study of this disease, specially considering the inclusion of the large number of patients and the long follow-up. In addition, it was performed in a third-level hospital setting, a reference institution in HL, where most clinical records are computerized, which implies a reliable record of the patients' medical history.

The interest of making future updates analyzing the hypotheses of this work is raised, since the development of new drugs, fundamentally based on anti-PD1 antibodies, such as nivolumab, has showed high response rates in adults with relapsed or refractory classical LH [12], which is expected to lead to a change in routine clinical practice.

Finally, this study is unique in the sense of analyzing the mortality of the patients comparing it to the Spanish population 40 years after diagnosis. Long-term follow-up 
of patients with HL may therefore focus on identifying late complications that constitute the leading cause of death in these patients.

\section{MATERIALS AND METHODS}

\section{Patients}

This study was performed at the Medical Oncology Department, at Puerta de Hierro University Hospital (Madrid, Spain). A total of 595 patients diagnosed with HL were included at our institution between January 1966 and February 2014. An anatomopathological confirmation of HL by a hematopathologist was considered mandatory for the inclusion of patients in the study.

The following variables were collected from the clinical records according to the standard hospital protocol: demographic data from each patient, clinical and pathological features, prognostic factors and treatments received type of response and survival, as well as the detection of specific causes of death.

All patients had to undergo an adequate extension study prior to initiation of treatment and during followup. In all cases, the stage was determined according to the Ann Arbor classification [13], later modified in 1988 at the Cotswolds meeting [14].

The following chemotherapy regimens were used: MOPP (clomiphene hydrochloride, vincristine sulfate, procarbazine hydrochloride and prednisone), ABVD (adriamycin, bleomycin, vinblastine and dacarbazine), a hybrid regimen of both, or other schemes in a small number of patients. Radiation therapy was performed following Kaplan's field recommendations [15].

Information on the progression of disease or relapse, re-treatment, and death, as well as its causes, was verified through the analysis of medical health records, death certificates, or National Institute of Statistics (INE) records. The cause of death associated with HL included progression of the disease independently of other causes of death. Non-HL related deaths were included in those causes that occurred independent of lymphoma, secondary to the toxicities of chemotherapy treatments and development of secondary malignancies.

The study was reviewed and approved by the Ethics and Clinical Research Committee of Puerta de Hierro University Hospital.

\section{Statistical methodology}

\section{Standardized mortality ratio analysis}

Data from the Spanish general population were provided by the National Center for Epidemiology and registered by the INE from 1980 to 2013. Therefore, patients diagnosed from January 1980 were selected for this analysis. Patients with follow-up after December 31 st 2013 were censored at this date. The standardized mortality ratio (SMR) is the ratio of the number of observed deaths (O) in our cohort versus expected (E) in the general Spanish population, adjusted for age, sex and time period. Both SMR and 95\% confidence intervals (CI) were estimated on the hypothesis basis of the Poisson distribution for deaths observed in the follow-up period $[16,17]$. Results were obtained for the entire cohort, segmented by gender, age groups at diagnosis $(\leq 30$, $31-59$, and $\geq 60$ years), and year of diagnosis $(<2000$ and $\geq 2000$ ).

\section{Survival analysis of the entire cohort}

All patients diagnosed with HL between 1966 and 2014 were included in this analysis. OS was defined as the time from diagnosis to the death of the patient; progression-free survival (PFS) was defined as the time from the end of treatment to relapse or death of the patient by any cause; and disease-free survival (DFS) as the time interval from first line treatment and a complete response, to relapse or death. If no events occured, observations were censored at the time of the last revision. The crude probability of the event was estimated using the KaplanMeier method, and the differences between the patient groups were assessed using the log-rank test.

A multivariate model of Cox proportional hazards was estimated, including sex, age at diagnosis (including three categories: $\leq 30$ years, $31-59$ years and $\geq 60$ years), and diagnostic period (before and after 2000) to establish their association together in OS. The assumption of proportional hazards was assessed using Schoenfeld's residues [18].

All statistical tests were two-tailed, and a $\mathrm{p}$ value $<0.05$ was considered statistically significant. All analyses were performed using Stata v14.2 (StataCorp, 2015. CollegeStation, TX: StataCorp LP).

\section{Author contributions}

All authors were responsible for the conception and design of the study and equally contributed to the elaboration of the present manuscript; all authors provided administrative support, provided study materials or patients, collected and assembled data, and approved the final manuscript.

This manuscript is not under consideration elsewhere.

\section{CONFLICTS OF INTEREST}

The authors declare no potential conflicts of interest.

\section{REFERENCES}

1. GLOBOCAN 2012. Estimated cancer incidence, mortality and prevalence worldwide in 2012. Internacional Agency 
for Research on Cancer, 2012. http://globocan.iarc.fr/Pages/ fact_sheets_population.aspx (Accessed on May 10 2017).

2. Rueda A. SEOM 2017. Linfoma de Hodgkin. Marzo 2017. http://www.seom.org/es/info-sobre-el-cancer/linfomahodgkin?start=1 (Accessed on May 10 2017).

3. REDECAN (Red Española de Registros de Cáncer). Estimaciones de la incidencia y la supervivencia del cáncer en España y su situación en Europa. Octubre 2014. http:// redecan.org/es/ page.cfm?id=196\&title=estimaciones-dela-incidencia-y-la-supervivencia-del-cancer-en-espana-ysu-situacion-en-europa (Accessed on May 10 2017).

4. Koshy M, Fairchild A, Son CH, Mahmood U. Improved survival time trends in Hodgkin'slymphoma. Cancer Med. 2016; 5:997-1003.

5. Sud A, Thomsen H, Sundquist K, Houlston RS, Hemminki K. Risk of Second Cancer in Hodgkin Lymphoma Survivors and Influence of Family History. J Clin Oncol. 2017; 35:1584-1590.

6. Canellos GP, Rosenberg SA, Friedberg JW, Lister TA, Devita VT. Treatment of Hodgkin lymphoma: a 50-year perspective. J Clin Oncol. 2014; 32:163-8.

7. Baxi SS, Matasar MJ. State-of-the-artissues in Hodgkin'slymphomasurvivorship. Curr Oncol Rep. 2010; 12:366-73.

8. Aleman BMP, van den Belt-Dusebout AW, Klokman WJ, Van't Veer MB, Bartelink H, van Leeuwen FE. Long-term cause-specific mortality of patients treated for Hodgkin's disease. J Clin Oncol. 2003; 21:3431-9.

9. Provencio M, García-López FJ, Bonilla F, España P. Comparison of the long-term mortality in Hodgkin's disease patients with that of the general population. Ann Oncol. 1999; 10:1199-205.

10. Kreissl S, Mueller H, Goergen H, Mayer A, Brillant C, Behringer K, Halbsguth TV, Hitz F, Soekler M, Shonukan O, Rueffer JU, Flechtner HH, Fuchs M, et al. Cancer-related fatigue in patients with and survivors of Hodgkin's lymphoma: a longitudinal study of the German Hodgkin Study Group. Lancet Oncol. 2016; 17:1453-62.

11. Best T, Li D, Skol AD, Kirchhoff T, Jackson SA, Yasui Y, Bhatia S, Strong LC, Domchek SM, Nathanson KL, Olopade OI, Huang RS, Mack TM, et al. Variants at 6q21 implicate PRDM1 in the etiology of therapy-induced second malignancies after Hodgkin's lymphoma. Nat Med. 2011; 17:941-3.

12. Younes A, Santoro A, Shipp M, Zinzani PL, Timmerman JM, Ansell S, Armand P, Fanale M, Ratanatharathorn V, Kuruvilla J, Cohen JB, Collins G, Savage KJ, et al. Nivolumab for classical Hodgkin's lymphoma after failure of both autologous stem-cell transplantation and brentuximab vedotin: a multicentre, multicohort, single-arm phase 2 trial. Lancet Oncol. 2016; 17:1283-94.

13. Carbone PP, Kaplan HS, Musshoff K, Smithers DW, Tubiana M. Report of the Committee on Hodgkin's disease staging classification. Cancer Res. 1971; 31:1860-1.

14. Lister TA, Crowther D, Sutcliffe SB, Glatstein E, Canellos GP, Young RC, Rosenberg SA, Coltman CA, Tubiana M. Report of a committee convened to discuss the evaluation and staging of patients with Hodgkin's disease: cotswolds meeting. J Clin Oncol. 1989; 7:1630-6.

15. Kaplan HS, Rosenberg SA. Extended field radical radiotherapy in advanced Hodgkin's disease. Cancer Res. $1966 ; 26: 1268-76$.

16. Therneau TM, Grambsch PM. Modeling Survival Data Extending the Cox Model. Springer; 2000; 261-287.

17. Breslow NE, Day NE. Statistical methods in cancer research. Volume II-The design and analysis of cohort studies. IARC Sci Publ. (1-406, 1987).

18. Grambsch PM, Therneau TM. Proportional hazards tests and diagnostics based on weighted residuals. Biometrika. 1994; 81:515-526. 